\title{
Bipolar radiofrequency ablation: the impact of tip load, application duration, power, and indifferent electrode size on the transmurality of a lesion
}

Artur Baszko ${ }^{1, A-C, F}$, Wojciech Telec ${ }^{1, B, E-F}$, Piotr Kałmucki ${ }^{1, A-C, F}$, Karol Kochman ${ }^{1, A-C, F}$, Izabela Miechowicz ${ }^{1, C-D, F}$, Stefan Ożegowski ${ }^{1, \mathrm{~B}-\mathrm{C}, \mathrm{F}}$, Andrzej Szyszka ${ }^{1, \mathrm{~A}, \mathrm{C}-\mathrm{F}}$

A - Research concept and design, B - Collection and/or assembly of data, C - Data analysis and interpretation,

D - Writing the article, E - Critical revision of the article, F - Final approval of article

1. Uniwersytet Medyczny im. K. Marcinkowskiego

Address for correspondence:

Artur Baszko, Uniwersytet Medyczny im. K. Marcinkowskiego email: abaszko@wp.pl

Wojciech Telec, Uniwersytet Medyczny im. K. Marcinkowskiego email: wojtek.telec@gmail.com

Piotr Kałmucki, Uniwersytet Medyczny im. K. Marcinkowskiego email: pkalm@poczta.fm

Karol Kochman, Uniwersytet Medyczny im. K. Marcinkowskiego email: kochman.karol@o2.pl

Izabela Miechowicz, Uniwersytet Medyczny im. K. Marcinkowskiego email: iza@ump.edu.pl

Stefan Ożegowski, Uniwersytet Medyczny im. K. Marcinkowskiego email: stefano@post.home.pl

Andrzej Szyszka, Uniwersytet Medyczny im. K. Marcinkowskiego email: andrzejszyszka@wp.pl

Received: 2019-01-02

Revised:

Accepted: 2019-01-07

Final review: 2019-01-07

DOI: $10.24255 / \mathrm{hbj} / 102680$

\section{Key words:}

bipolar radiofrequency ablation, active and ground electrodes, ventricular tachycardia, scar formation

\section{Abstract}

\section{Background}

The main determinant of the radiofrequency (RF) ablation effect is transmurality of the lesion. Bipolar radiofrequency ablation (BA) creates transmurality in a higher proportion of cases than unipolar ablation (UA), but little is known about optimal RF settings.

\section{Material and methods}

This study aimed to compare UA and BA based on different application time, cathode tip load, anode size and power settings for achieving a transmural lesion in vitro. A Thermocool electrode was used for ablation (cathode) and a $4 \mathrm{~mm}$ or $8 \mathrm{~mm}$ tip catheter was used as the ground electrode (anode) for BA. The tested settings were tip load (10 $\mathrm{g}$ and $20 \mathrm{~g}$ ), power (30 W or $40 \mathrm{~W}$ ), and application duration (30, 60 , or 90 s). After ablation, we measured scar size and transmurality of the lesion.

\section{Results}

BA created significantly larger and deeper scars compared with UA. Transmurality was achieved respectively in $42 \%$ and $2 \%$ of cases $(\mathrm{p}<0.001)$. Higher tip load resulted in larger scar size, and transmurality was achieved in $37 \%$ and $20 \%$ of cases $(\mathrm{p}=0.05)$. Scar size and transmurality increased with RF duration (5\%, 39\% and $42 \%$ of specimens, respectively $(\mathrm{p}<0.001)$. Bigger tip size of the ground electrode was related to higher transmurality ( $22 \%$ vs $61 \%$, $\mathrm{p}<0.001)$. No relationship was found between power and transmurality. Optimal parameter settings were $20 \mathrm{~g}$ tip load, indifferent electrode with $8 \mathrm{~mm}$ tip, and $90 \mathrm{~s}$ RF duration (97.6\% transmurality).

\section{Conclusions}

BA is more effective than UA in achieving transmural lesions. Optimal settings for BA consist of longer RF duration, higher tip load, and bigger tip size of the indifferent electrode. 
Developments in three-dimensional scar visualization based on magnetic resonance imaging (MRI) and electroanatomical mapping techniques influence our understanding of the mechanisms of ventricular tachycardia $(\mathrm{VT})^{(1)}$. This has led to substantial evolution of the VT ablation technique from the classical method, based on entrainment, to substrate modification. With this approach the success rate of ablation has increased substantially over the years ${ }^{(2),(3)}$. However, there remain cases of VT with a reentrant circuit located deep in the myocardium, mainly in the interventricular septum ${ }^{(4)}$. VT ablation in this group is challenging as the thickness of the muscle prevents transmurality with unipolar radiofrequency (RF) ablation, even if delivered sequentially from both sides of the myocardium ${ }^{(5),(6)}$. Recently there have been several reports on bipolar ablation in patients with atrial flutter and ventricular tachycardia resistant to conventional ablation techniques ${ }^{(7),(8)}$. The delivery of RF current between two electrodes located on the opposite sides of the muscle increases current density and may result in a higher proportion of transmurality. In in vitro studies, bipolar RF ablation generally created larger lesions than unipolar RF ablation. However, there are conflicting results with respect to optimal RF settings and the position of the electrodes to obtain transmurality $(7),(9),(10)$.

The aim of our study was to compare unipolar (UA) and bipolar ablation (BA), based on different procedural parameter settings, for in vitro porcine specimens of the interventricular septum. The purpose of the study was to establish the most successful and safe parameter combinations based on RF duration, power, contact force, and the dispersive electrode size.

\section{Methods}

The study protocol was in accordance with guidelines for care and treatment of experimental animals of Poznan University of Medical Sciences. The interventricular septum was isolated from a fresh porcine heart and the septum was cut into approximately $15 \mathrm{~mm}$-thick slabs. These were then immersed in a single bath at a temperature of $37^{\circ} \mathrm{C}$, with constant flow of isotonic water solution and the impedance between 120 and $140 \Omega$. The active ablation catheter was a $3.5 \mathrm{~mm}$ irrigated tip Thermocool EzSteer electrode (Biosense Webster, Diamond Bar, CA, USA), which was placed perpendicular to the myocardial surface. The tip load of the active electrode was modified using lead blocks attached to the vertically aligned catheters. Celsius catheters with 4-mm and 8-mm tips (Biosense Webster, Diamond Bar, CA, USA) were used as the ground electrodes for BA, positioned parallel to the tissue surface, and were connected with the indifferent electrode receptacle of the RF generator (Stockert) using a custom-made connector. Ablation was performed in power controlled mode with 25 $\mathrm{mL} / \mathrm{min}$ saline flow, with the catheter tip temperature limited to $44^{\circ} \mathrm{C}$, using different tip loads (10 $\mathrm{g}$ and $20 \mathrm{~g}$ ), power outputs $(30 \mathrm{~W}, 40 \mathrm{~W})$, application duration (30, 60, and $90 \mathrm{~s})$, and size of the ground electrode tip ( $4 \mathrm{~mm}$ and $8 \mathrm{~mm}$ ). During the application, impedance and steam pop were recorded. If steam pop occurred, the application was terminated and the time was recorded. For any combination of RF settings, three applications were done. Following the ablation, the tissue block was removed from the bath and marked with a code to blind the parameter settings. Direct measurements were performed by a person who was blinded to the RF settings. The "entry" of the RF lesion was at the site of the active ablation location, while the "exit" was at the opposite site, where the ground pad (UA) or ground electrode was positioned (BA). The following parameters were recorded: slab thickness, entry (D_En) and exit (D_Ex) scar diameters, the total scar depth, and the presence of a transmural scar. From the depth and diameter, the volume of the scar was calculated based on the assumption that the scar is of truncated cone shape.

$$
\left(V=\frac{P i}{3} * h *\left(R^{2}+R * r+r^{2}\right) .\right.
$$

Where: $\mathrm{V}=$ volume, $\mathrm{h}=$ depth, $\mathrm{R}, \mathrm{r}=$ radius of entry and exit

\section{Statistics}

Statistical analyses were performed using statistical software (Statistica v.12, StatSoft, Krakow, Poland). A P value of less than 0.05 was considered statistically significant. Bonferroni correction was applied when analyzing only selected groups. Categorical variables were expressed as numbers and percentages. Continuous data were checked for normal distribution by the Shapiro-Wilk test and presented as the means and standard deviations (SDs). Univariate analyses were performed. Categorical variables were analyzed by the $\chi 2$ test or Fisher's exact test, when applicable. Comparisons of continuous data with normal distribution were performed using Student's t-test or the Cochran-Cox test. Comparisons of continuous data, which were non-normally distributed, were performed using the Mann-Whitney U test. Logistic regression (stepwise forward logistic regression) was performed to find variables which are the most important in predicting transmurality. Odds ratios (ORs) and 95\% confidence intervals (CIs) were estimated for statistically significant variables. The Wald test was used to test the overall contribution of variables to the model.

\section{Results}

Comparison between unipolar and bipolar RF energy based on scar size and transmurality

The comparison between unipolar and bipolar ablation at different RF settings is presented in Figure 1. Bipolar ablation

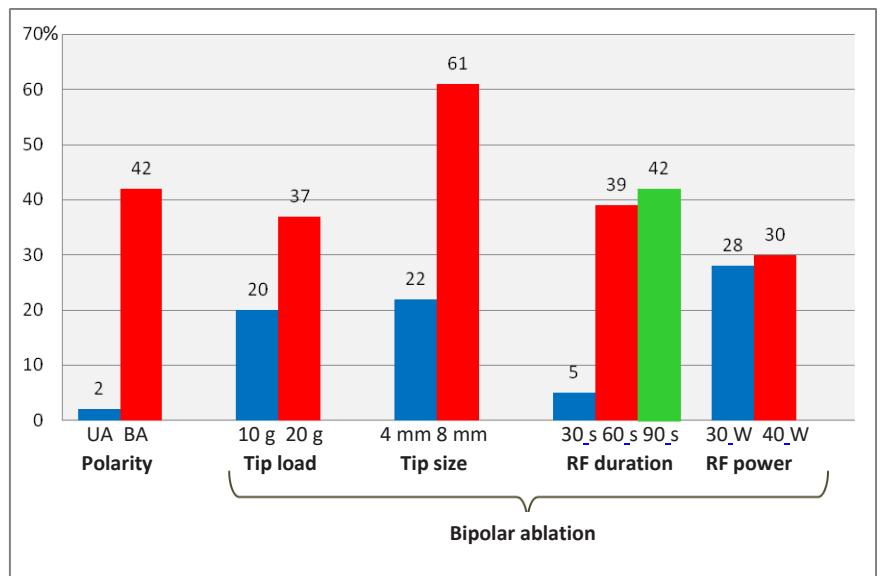

Figure1. Transmurality in the unipolar (UA) and bipolar (BA) ablation with $4 \mathrm{~mm}$ and 8 $\mathrm{mm}$ tip ground electrode, different application durations, and RF power 
created larger and deeper scars than UA did for all settings: D_En $7.5 \pm 3.4 \mathrm{~mm}$ vs. $8.1 \pm 2.4 \mathrm{~mm}(\mathrm{p}=0.4)$, D_Ex $8.0 \pm 2.4 \mathrm{~mm}$ vs. $0.4 \pm 1.3 \mathrm{~mm}(\mathrm{p}<0.001)$, and total depth was $11.4 \pm 3.4 \mathrm{~mm}$ vs. $7.2 \pm 2.2 \mathrm{~mm}(\mathrm{p}<0.001)$. The scar volume was significantly larger in bipolar ablation (1074 \pm 971 vs. $162 \pm 147 \mathrm{~mm} 3, \mathrm{p}<0.001)$. Transmurality was achieved in $2 \%$ of unipolar and $42 \%$ of bipolar RF applications $(\mathrm{p}<0.001)$.
Effect of ablation electrode tip load, ground electrode tip size, RF power, and duration for scar formation

There was a significant correlation between parameters used during ablation and the transmurality and pop occurrence in bipolar ablation. Higher tip load resulted in a larger scar entry diameter and scar depth. The scar exit diameter was similar in both groups, with $10 \mathrm{~g}$ and $20 \mathrm{~g}$ electrode tip loads (Table 1). The percentage of scarring (scar/tissue ratio) was significantly higher for the $20 \mathrm{~g}$ electrode tip load. Trans-

Table 1. Relationship between tip load of the ablation electrode and scar size parameters.

\begin{tabular}{|l|l|l|l|}
\hline \multirow{2}{*}{ Parameter } & \multicolumn{2}{|c|}{ Ablation electrode tip load } \\
\cline { 2 - 4 } & $10 \mathrm{~g}(\mathrm{n}=36)$ & $20 \mathrm{~g}(\mathrm{n}=36)$ \\
\hline Entry diameter $(\mathrm{mm})$ & $6.2 \pm 3.7$ & $8.9 \pm 2.7$ & 0.0007 \\
\hline Exit diameter $(\mathrm{mm})$ & $8.1 \pm 2.3$ & $7.9 \pm 2.5$ & $10.7 \pm 4.8$ \\
\hline Entry depth $(\mathrm{mm})$ & $7.8 \pm 5.2$ & $9.9 \pm 5.6$ & 0.73 \\
\hline Exit depth $(\mathrm{mm})$ & $6.6 \pm 5.3$ & $12.4 \pm 3.0$ & 0.0162 \\
\hline Total depth $(\mathrm{mm})$ & $10.5 \pm 3.5$ & $1401.8 \pm 1034.4$ & 0.0126 \\
\hline Scar volume $(\mathrm{mm})$ & $738.6 \pm 782.6$ & $83.3 \pm 20.8$ & 0.0033 \\
\hline Scar/tissue ratio $(\%)$ & $65.9 \pm 24.0$ & & 0.0015 \\
\hline
\end{tabular}

murality was achieved in $20 \%$ of specimens with $10 \mathrm{~g}$ and in $37 \%$ with $20 \mathrm{~g}$ tip pressure $(\mathrm{p}=0.05)$.
The RF duration was significantly related to differences in most analyzed parameters (Table 2). There was a significant difference between the results obtained with $30 \mathrm{~s}$ application

Table 2. Comparison between RF duration and scar size parameters..

\begin{tabular}{|c|c|c|c|c|c|}
\hline \multirow{2}{*}{ Parameter } & \multicolumn{3}{|c|}{ RF duration } & \multirow{2}{*}{$\begin{array}{c}\text { P value } \\
(30 \text { vs } 60)\end{array}$} & \multirow{2}{*}{$\begin{array}{c}P \text { value } \\
(30 \text { vs } 60)\end{array}$} \\
\hline & $30 s(n=24)$ & $60 s(n=24)$ & $90 s(n=24)$ & & \\
\hline Entry diameter (mm) & $5.8 \pm 2.6$ & $8.5 \pm 3.9$ & $8.3 \pm 3.2$ & 0.008 & 0.87 \\
\hline Exit diameter (mm) & $7.3 \pm 2.7$ & $8.5 \pm 1.9$ & $8.2 \pm 2.3$ & 0.09 & 0.68 \\
\hline Entry depth (mm) & $6.0 \pm 3.7$ & $10.3 \pm 5.6$ & $11.5 \pm 4.5$ & 0.003 & 0.42 \\
\hline Exit depth (mm) & $4.3 \pm 3.7$ & $10.1 \pm 5.6$ & $10.5 \pm 5.4$ & 0.0001 & 0.78 \\
\hline Total depth (mm) & $9.1 \pm 2.5$ & $12.3 \pm 3.7$ & $13.0 \pm 2.6$ & 0.0009 & 0.44 \\
\hline Scar volume (mm3) & $454.6 \pm 544.8$ & $1444.9 \pm 1034.5$ & $1335.9 \pm 966.4$ & 0.0001 & 0.71 \\
\hline Scar/tissue ratio \% & $59.1 \pm 17.7$ & $79.8 \pm 25.3$ & $85.0 \pm 20.8$ & 0.002 & 0.43 \\
\hline
\end{tabular}

Data are mean $\pm \mathrm{SD}$.

time and both $60 \mathrm{~s}$ and $90 \mathrm{~s}$. However, no significant gain was achieved when application duration was extended from $60 \mathrm{~s}$ to $90 \mathrm{~s}$. The percentage of scarring (scar/tissue ratio) was significantly higher with longer application duration. Transmurality was achieved in $5 \%, 39 \%$ and $42 \%$ of specimens respectively $(\mathrm{p}<0.001)$.

The tip size of the ground electrode was related to a higher scar/tissue ratio and transmurality (Table 3). There was no difference in D_En and D_Ex, but scar depth was significantly larger when the $8 \mathrm{~mm}$ tip electrode was used $(12.3 \pm 3.3 \mathrm{vs}$. $10.6 \pm 3.2 \mathrm{~mm}, \mathrm{p}=0.02)$. Transmurality was obtained in $22 \%$ of cases with $4 \mathrm{~mm}$ and in $61 \%$ of cases with $8 \mathrm{~mm}$ ground tip electrode $(p<0.001)$.

Ablation was performed using $30 \mathrm{~W}$ and $40 \mathrm{~W}$ power. The power change had generally no effect on the scar size param- 
Table 3. Comparison between ground electrode tip size and scar size parameters.

\begin{tabular}{|l|l|l|l|}
\hline \multirow{2}{*}{ Parameter } & \multicolumn{2}{|c|}{ Ground electrode tip size } \\
\cline { 2 - 4 } & $\mathbf{4} \mathbf{m m}(\mathbf{n = 3 6 )}$ & $\mathbf{8} \mathbf{~ m m}(\mathbf{n = 3 6 )}$ \\
\hline Entry diameter $(\mathrm{mm})$ & $6.7 \pm 3.8$ & $8.3 \pm 2.9$ & 0.05 \\
\hline Exit diameter $(\mathrm{mm})$ & $8.2 \pm 2.4$ & $7.8 \pm 2.4$ & 0.52 \\
\hline Entry depth $(\mathrm{mm})$ & $7.3 \pm 4.9$ & $11.2 \pm 4.8$ & 0.001 \\
\hline Exit depth $(\mathrm{mm})$ & $6.4 \pm 4.8$ & $10.1 \pm 5.8$ & 0.005 \\
\hline Total depth $(\mathrm{mm})$ & $10.6 \pm 3.3$ & $12.3 \pm 3.3$ & $<0.03$ \\
\hline Scar volume $(\mathrm{mm})$ & $831.3 \pm 983.1$ & $83.2 \pm 23.6$ & $<0.04$ \\
\hline Scar/tissue ratio $\%$ & $66.2 \pm 21.6$ & & 0.002 \\
\hline
\end{tabular}

Data are mean \pm SD.

eters (except scar entry diameter, $\mathrm{p}=0.01$ ) or transmurality (respectively $30 \%$ vs. $28 \%$, p=ns).

Steam pop occurred only once in UA. It was recorded in 27 seconds of application with maximal ablation settings ( $20 \mathrm{~g}$ tip load and power of $40 \mathrm{~W}$ ). It was not related to tissue disruption or transmurality. In fact, the scar/tissue ratio was $67 \%$. There was no significant change in impedance before steam pop $(199 \Omega$ to $95 \Omega)$. In bipolar ablation, steam pop was more common $(n=9)$. It occurred after a mean of 43 seconds of application (range 22 seconds to 85 seconds) and was not related to significant visual tissue damage. It was significantly more common when an $8 \mathrm{~mm}$ indifferent electrode was used, with higher tip load and power, but never resulted in perforation. In the steam pop group all of the analyzed scar parameters were significantly higher (scar depth $14.3 \pm 2.1 \mathrm{~mm}$ vs. $11.1 \pm 3.3$ $\mathrm{mm}, \mathrm{p}=0.005$, scar volume $2319.8 \pm 1015.8 \mathrm{~mm} 3$ vs. $894.1 \pm 828.2$

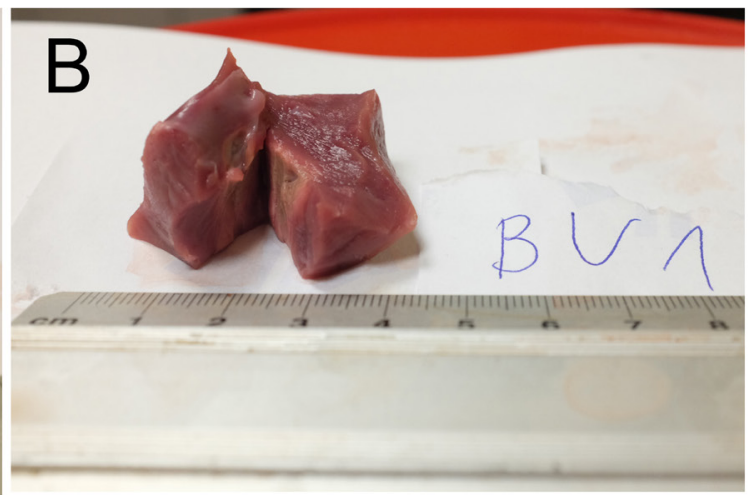

BA, 20g CF, $60 \mathrm{sec}, 30$ Watt, $8 \mathrm{~mm}$ tip GE

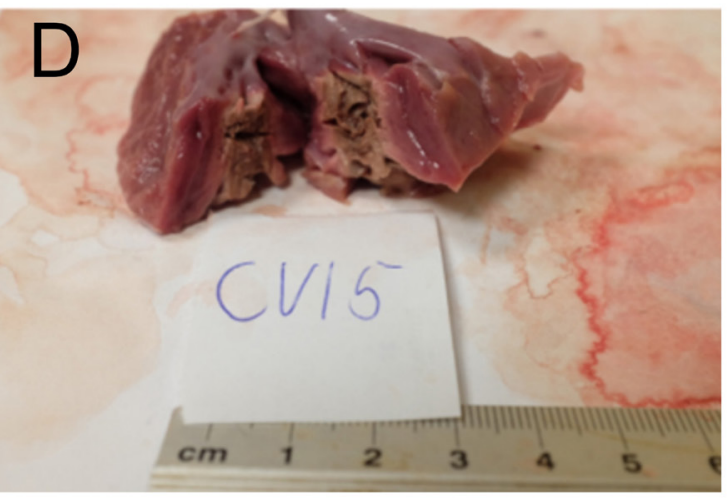

BA, $20 \mathrm{~g} \mathrm{CF}, 90 \mathrm{sec}, 40$ Watt, $4 \mathrm{~mm}$ tip GE 
$\mathrm{mm} 3, \mathrm{p}<0.0001$ and scar/tissue ratio $95.6 \pm 13.3 \%$ vs. $71.6 \pm$ $23.7 \%, \mathrm{p}=0.004)$. In the group with steam pop, transmurality was present in $27 \%$. Figure 2 presents the images of lesions following applications.

The baseline impedance for UA was $117 \pm 21 \Omega$ and $113 \pm 20$ $\Omega$ for BA ( $\mathrm{p}=0.3$ ). The impedance was dropped to $79 \pm 13 \Omega$ for UA and $88 \pm 14 \Omega$ for $B A(p=0.002)$. There were no significant differences in impedance drop in transmural ablation $(30.4 \pm 11.0$ vs. $30.8 \pm 18.1 \Omega$ ) or when pop occurred (25.5 \pm 2.1 vs. $30.9 \pm 16 \Omega$ ).

Regression analysis was used to identify the best parameter settings predicting transmurality. The $20 \mathrm{~g}$ tip load, 8 $\mathrm{mm}$ tip indifferent electrode, and $90 \mathrm{~s}$ RF duration resulted in $97.6 \%$ transmurality. If RF duration was reduced to $60 \mathrm{~s}$, transmurality was obtained in $85.7 \%$. The lowest probability of transmurality was observed for $30 \mathrm{~s}$ application with a 10 $\mathrm{g}$ tip load and $4 \mathrm{~mm}$ indifferent electrode size (6.5\%). The smaller ground electrode required a higher tip load and longer application time to obtain a higher $(66.7 \%)$ probability of transmurality ( $4 \mathrm{~mm}, 20 \mathrm{~g}, 90 \mathrm{~s})$. The $30 \mathrm{~s}$ application duration was too short to obtain transmurality in most of the settings.

\section{Discussion}

The most important result of our study is that bipolar RF ablation creates bigger lesions and is by far more successful in obtaining transmurality of the lesion than unipolar ablation. We also proposed the most effective configuration of bipolar RF ablation. During a standard ablation, RF energy is delivered between the tip of the electrode and the dispersive pad located under the patient. The electrical alternant current flowing through the tissue encounters resistance and the energy is converted into heat. This energy, resistive heat, coagulates the tissue close to the electrodes. The heat is then transmitted to the surrounding tissue by conduction and radiation. Both types of injuries created by resistive heat and convection are responsible for lesion formation. If the distance between the tip of the ablation electrode and the ground electrode is decreased, it will result in higher current density with more resistive heating, leading to deeper lesion formation. This is the concept behind bipolar RF ablation. It has also been well documented that scar formation is related to the electrode size, the energy power, the contact force between the electrode and the tissue, and the cooling effect of surrounding fluid, and only a small part of the energy is delivered to the tissue ${ }^{(11)}$. The concern that high power bipolar ablation between two closely located electrodes can result in large and uncontrolled lesions has not been supported by in vitro experiments. In fact, the lesions created by bipolar ablation are narrower and deeper, causing transmural scarring in tissue as thick as $25 \mathrm{~mm}^{(5),(9)}$. With good control of the impedance and contact pressure the bipolar source of RF energy seems to be safe, as confirmed by others ${ }^{(10)}$. Unfortunately, there are no commercially available tools and connectors available for use during these procedures, and they must be customized.

There are several clinical studies showing that bipolar ablation is effective in supraventricular and ventricular arrhythmia, but from these studies it is evident that there are no generally accepted settings for applications. In a case of idiopathic VT described by Merino et al., RF energy was delivered between two non-irrigated electrodes located in the aortic sinus of Valsalva and the left ventricle ${ }^{(13)}$. Gizurarson et al. used irrigated electrodes with a flow of $30 \mathrm{~mL} / \mathrm{min}, 40 \mathrm{~W}$ power, and a $65^{\circ} \mathrm{C}$ temperature $\operatorname{limit}^{(8)}$. In the experimental part of their study, Koruth at al. used two irrigated $3.5 \mathrm{~mm}$ tip electrodes connected to an RF generator with a flow of $30 \mathrm{~mL} / \mathrm{min}^{(9)}$. The 30-50 W power setting was used and constant pressure was maintained for $120 \mathrm{~s}$ of application. They obtained $82 \%$ transmural lesions in a series of 28 specimens of $17.4 \pm 3.3 \mathrm{~mm}$ thickness. Bipolar lesions were present in $100 \%$ of $10-15 \mathrm{~mm}$, $89 \%$ of $15-20 \mathrm{~mm}, 66.7 \%$ of $20-25 \mathrm{~mm}$, and $0 \%$ of $25-30 \mathrm{~mm}$ thick specimens. In the latter subgroup, there was only one case. In the clinical part of their study, the mean time to terminate VT was $27 \pm 5$ seconds. Sauer et al. presented a case of septal VT ablation where an $8 \mathrm{~mm}$ electrode was used as the active electrode and a $3.5 \mathrm{~mm}$ irrigated tip as the ground tip, connected to an RF generator with a power limit of $70 \mathrm{~W}$ (14). We recently described the case of a patient with hypertensive cardiomyopathy and septal VT who underwent 4 unsuccessful $\mathrm{RF}$ ablations ${ }^{(12)}$. We used $30 \mathrm{~W}$ power, $25 \mathrm{~mL} / \mathrm{minute}$ flow, and a $43^{\circ} \mathrm{C}$ temperature limit. The bipolar application was successful and terminated incessant tachycardia in $72 \mathrm{~s}$. From these studies, it is evident that numerous different settings have been clinically tested and proven to be successful, but no uniform recommendations were established.

In most clinical situations when we deal with complex arrhythmia, we can adjust several RF parameters to obtain the most effective lesion formation including the type and size of the electrodes, RF power, duration of application, and contact force between electrode and tissue. Several of those parameters were tested in vitro in various configurations. Nagashima et al. compared unipolar RF with bipolar RF ablation using $4 \mathrm{~mm}$ tip irrigated electrodes $(20 \mathrm{~mL} / \mathrm{min}$ irrigation rate) delivered in a single application lasting $120 \mathrm{~s}$ with 30, 50, and $70 \mathrm{~W}$ energy $^{(5)}$. Transmurality was achieved with bipolar ablation in a higher proportion of interventricular swine specimens for different power settings in 50\%, $46 \%$, and $71 \%$ respectively. In this experiment, they did not record contact force. Ikeda et al. performed a meticulous analysis of different contact force settings in bipolar ablation in beating dog hearts (15). They used low ( $8 \mathrm{~g})$, moderate $(21 \mathrm{~g})$, and large $(60 \mathrm{~g})$ contact force measured by a TactiCath electrode (St. Jude Medical) and two different powers of $30 \mathrm{~W}$ and $40 \mathrm{~W}$ for 60 seconds. For the low contact force, the higher the power was, the deeper and wider was the lesion. However, for a higher contact force there were no differences in lesion size for either 30 or $40 \mathrm{~W}$ of power. In this study, transmurality of the lesion was not assessed; only the lesion size was assessed. Nguyen et al. in a recent study investigated variable configurations during RF bipolar ablation, namely: tip size of active and ground electrodes (3.5 $\mathrm{mm}, 4 \mathrm{~mm}, 8 \mathrm{~mm}$ ), flow $30 \mathrm{~mL} / \mathrm{min}, 42^{\circ} \mathrm{C}$ temperature limit for irrigated electrodes, and parallel vs. perpendicular orientation of electrodes with respect to tissue surface ${ }^{(10)}$. The energy was titrated from 30 to $50 \mathrm{~W}$ observing the impedance. The RF application was terminated after $60 \mathrm{~s}$ or earlier when there 
was pop or abrupt increase or decrease in impedance. They documented that for all settings the perpendicular orientation of irrigated electrodes resulted in deeper and larger volume of the scar than a parallel position. In the case of an $8 \mathrm{~mm}$ tip ground electrode, the parallel orientation was related to larger scars. This was also confirmed by our study. We used $4 \mathrm{~mm}$ and $8 \mathrm{~mm}$ ground electrodes positioned parallel to the tissue while the active electrode was perpendicular, as this is the most typical approach in clinical situations. The $8 \mathrm{~mm}$ ground electrode resulted in significantly larger scars (entry, exit, depth, and volume). The $8 \mathrm{~mm}$ ground tip electrode was also related to significantly higher scar/tissue ratio $(66.2 \%$ vs. $88.2 \%$ ) and transmurality (61\% vs. $22 \%$ ).

Another important issue is the relationship between tissue thickness and the size of the lesion. Nguyen et al. found that there were no differences between unipolar and bipolar ablation when the tissue was $20 \mathrm{~mm}$ thick ${ }^{(10)}$. This is contrary to the results of the study by Koruth et al., where $66.6 \%$ of specimens of 20-25 mm thickness were transmural ${ }^{(9)}$. The only rational explanation could be the duration of the application, which was two times longer in their studies (120 s vs. 60 s). Also in our study, the longer duration of the application resulted in larger scars and a higher proportion of transmurality.

Because most ablations in VT related to structural heart disease require approximately linear application across the substrate, an important factor is the minimum duration of application to obtain transmurality. We added very important issues to the discussion about optimal settings for bipolar ablation. From many different configurations, we showed that the minimum duration of RF application had to be $60 \mathrm{~s}$. Application of $30 \mathrm{~s}$ resulted in only $5 \%$ lesion transmurality. While longer application time (90 s) did not result in a significantly larger scar size in comparison to application for 60 $\mathrm{s}$, longer time slightly improved transmurality. The longer application time may be necessary when the myocardium is thicker than $20 \mathrm{~mm}$, as discussed earlier.

It is also important to note that scar entry and exit sizes tend to be similar with longer application time, larger ground electrode size, and higher tip load, with the only difference being evident in lesion depth and scar volume.

Another important observation was that the increase in power did not result in a greater scar size or a higher transmurality rate. This observation is consistent with that of Ikeda et al., who also documented that, for a lower contact force (8 $\mathrm{g})$, the increase in energy resulted in a larger lesion, while for a higher contact force no such correlation was observed ${ }^{(15)}$. Nguyen et al. also tested high RF power $(70 \mathrm{~W})$, but only with an $8 \mathrm{~mm}$ tip active electrode, and observed that higher energy did not result in a larger lesion size but resulted in a significantly higher number of steam pops ${ }^{(10)}$. In our study, steam pop was more likely to occur in bipolar than unipolar ablation. Similarly to other studies, we found that steam pops occurred more frequently with higher RF energy and contact force. We also found a significant relationship between the ground electrode size and occurrence of steam pops. None of the previous studies analyzed the morphology of the lesion and transmurality in the steam pop group. We documented that steam pop does not necessarily indicate that the lesion is transmural, but the lesions are larger. In fact, transmurality was documented in only $27 \%$ of cases with steam pops. In cases when steam pop occurred, the application was stopped prematurely (mean time $43 \mathrm{~s}$ ), which in our opinion could be too short to obtain transmurality, which required longer application times. In the group with steam pop there was a significantly larger scar volume and a higher scar/tissue ratio. In the single steam pop case in unipolar ablation with $40 \mathrm{~W}$ and $20 \mathrm{~g}$ contact force, transmurality was not achieved, but the scar constituted $67 \%$ of the specimen thickness. In bipolar ablation without the pop, the scar/tissue ratio was $71.6 \%$ vs. $95.6 \%$ when the pop occurred. Based on these findings, it is advisable to stop application as steam pop occurs but continue the ablation thereafter, as the lesion may not be transmural.

\section{Study limitations}

The main limitation of the study was the use of tissue specimens of similar thickness. We used approximately 15 $\mathrm{mm}$ thick interventricular slabs. As there are concerns about the effect of bipolar ablation of tissue thicker than $20 \mathrm{~mm}$, the results should be viewed with caution. We performed only visual measurements of the scars. A microscopic evaluation of the scar could give more precise data on the scar size and morphology. Another important point is that the experiment was performed in saline solution, not on beating hearts. The solution in which specimens were immersed has different characteristics than the blood in in vivo situations, and this should be taken into consideration when applying the results to clinical situations.

\section{Clinical implications}

Irrigated bipolar RF ablation is superior to unipolar ablation in achieving transmurality at all typical RF settings. For irrigated bipolar ablation, the combination of RF duration (60 s or $90 \mathrm{~s}$ ), tip load (20 g), and $8 \mathrm{~mm}$ tip of the ground electrode results in the highest probability of transmurality, with little influence of increasing the power. Steam pop is related to larger scar size but does not indicate transmurality in most cases.

\section{References}

1. Andreu D, Berruezo A, Ortiz-Pérez JT et al., Integration of 3D electroanatomic maps and magnetic resonance scar characterization into the navigation system to guide ventricular tachycardia ablation. Circ Arrhythm Electrophysiol. 2011 Oct 1;4(5):674-83.

2. Silberbauer J, Oloriz T, Maccabelli G et al., Noninducibility and late potential abolition a novel combined prognostic procedural end point for catheter ablation of postinfarction ventricular tachycardia. Circ Arrhythm Electrophysiol. 2014 Jun 1;7(3):424-35.

3. Berruezo A, Fernández-Armenta J, Andreu D et al., Scar dechanneling: new method for scar-related left ventricular tachycardia substrate ablation. Circ Arrhythm Electrophysiol. 2015 Apr 1;8(2):326-36. 
4. Havranek S, Palecek T, Kovarnik T, et al., Arrhythmogenic substrate at the interventricular septum as a target site for radiofrequency catheter ablation of recurrent ventricular tachycardia in left dominant arrhythmogenic cardiomyopathy. BMC Cardiovascular Disorders (2015) 15:18 DOI 10.1186/s12872-015-0010-8

5. Nagashima K, Watanabe I, Okumura Y, et al., Lesion formation by ventricular septal ablation with irrigated electrodes: comparison of bipolar and sequential unipolar ablation. Circulation Journal 2011;75(3):565-70.

6. Yamada T, Maddox WR, McElderry HT, et al., Radiofrequency catheter ablation of idiopathic ventricular arrhythmias originating from intramural foci in the left ventricular outflow tract efficacy of sequential versus simultaneous unipolar catheter ablation. Circ Arrhythm Electrophysiol. 2015 Apr 1;8(2):344-52.

7. Piers SRD, Dyrda K, Tao Q, et al., Bipolar ablation of ventricular tachycardia in a patient after atrial switch operation for dextro-transposition of the great arteries. Circ Arrhythm Electrophysiol. 2012 Apr 1;5(2):e38-40.

8. Gizurarson S, Spears D, Sivagangabalan G, et al., Bipolar ablation for deep intra-myocardial circuits: human ex vivo development and in vivo experience. Europace 2014 16(11):1684-8.

9. Koruth JS, Dukkipati S, Miller MA, et al., Bipolar irrigated radiofrequency ablation: a therapeutic option for refractory intramural atrial and ventricular tachycardia circuits. Heart Rhythm. 2012 (12):1932-41.

10. Nguyen DT, Tzou WS, Brunnquell M, et al., Clinical and biophysical evaluation of variable bipolar configurations during radiofrequency ablation for treatment of ventricular arrhythmias. Heart Rhythm 2016 13: 2161-2171;

11. Wittkampf FH, Nakagawa H. RF Catheter ablation: lessons on lesions. Pacing Clin Electrophysiol. 2006 Nov 1;29(11):1285-97.

12. Baszko A, Telec W, Kałmucki P, et al., Bipolar irrigated radiofrequency ablation of resistant ventricular tachycardia with a septal intramural origin: the initial experience and a description of the method. Clin Case Rep. 2016 Aug 25;4(10):957-61.

13. Merino JL, Peinado R, Ramirez L, et al., Ablation of idiopathic ventricular tachycardia by bipolar radiofrequency current application between the left aortic sinus and the left ventricle. Europace. 2000 Oct 1;2(4):350-4.

14. Sauer WH, Steckman DA, Zipse MM, et al., High-power bipolar ablation for incessant ventricular tachycardia utilizing a deep midmyocardial septal circuit. Heart Rhythm Case Rep. 2015 Nov 1;1(6):397-400.

15. Ikeda A, Nakagawa H, Lambert H, et al., Relationship Between catheter contact force and radiofrequency lesion size and incidence of steam pop in the beating canine heart. Circ Arrhythm Electrophysiol. 2014 Dec 1;7(6):1174-80. 